\title{
Next-to-leading order $Q^{2}$-evolution of the transversity distribution $h_{1}\left(x, Q^{2}\right)$
}

\author{
A. Hayashigaki, Y. Kanazawa and Yuji Koike \\ Graduate School of Science and Technology, Niigata University, Ikarashi, Niigata 950-21, Japan
}

\begin{abstract}
We present a calculation of the two-loop anomalous dimension for the transversity distribution $h_{1}\left(x, Q^{2}\right), \gamma_{n}^{h(1)}$, in the MS scheme of the dimensional regularization. Due to the chiral-odd nature, $h_{1}$ does not mix with the gluon distributions, and thus our result is the same for the flavor-singlet and nonsinglet distributions. At small $n$ (moment of $h_{1}$ ), $\gamma_{n}^{h(1)}$ is significantly larger than $\gamma_{n}^{f(1)}$ (the anomalous dimension for the nonsinglet $f_{1}$ ), but approaches $\gamma_{n}^{f(1)}$ very quickly at large $n$, keeping the relation $\gamma_{n}^{h(1)}>\gamma_{n}^{f(1)}$. This feature is in parallel to the relation between the one-loop anomalous dimension for $f_{1}$ and $h_{1}$.
\end{abstract}

PACS numbers: 13.85.Qk, 12.38.Bx, 13.88.+e, 14.20.Dh 


\section{Introduction}

It is well known that the nucleon (spin-1/2 hadron) has three independent twist-2 quark distributions $f_{1}\left(x, Q^{2}\right), g_{1}\left(x, Q^{2}\right)$ and $h_{1}\left(x, Q^{2}\right)$. These distributions have a simple parton model interpretation and appear as a leading contribution to various hard (semi-) inclusive processes. The first two distributions, $f_{1}$ and $g_{1}$, are chiral-even and can be measured through the totally inclusive deep inelastic lepton-nucleon scattering (DIS). In fact, accumulated DIS data on $f_{1}$ and $g_{1}$ has provided us with a rich information about the parton distributions in the nucleon. The third one, $h_{1}$, is chiral-odd and cannot be measured through the totally inclusive DIS. However, it appears as a leading contribution to the transversely polarized nucleon-nucleon Drell-Yan process 1, 2, 3, 61, semi-inclusive productions of baryons and pion in the DIS [2, 3], etc. This spin-dependent quark distribution, named as transversity distribution in [3], measures the probability in a transversely polarized nucleon to find a quark polarized parallel to the nucleon spin minus the probability to find it oppositely polarized. It thus supplies an information on the nucleon's spin structure not provided by the $g_{1}$ distribution, and is expected to be measured by RHIC in the near future.

The $Q^{2}$-evolution of these parton distribution functions is calculable in perturbative QCD. It is governed by the anomalous dimensions for the corresponding twist-2 operators and the $\beta$-function. Leading order (LO) $Q^{2}$-evolution of $f_{1}$ and $g_{1}$ has been known for a long time [5] and the LO $Q^{2}$-evolution for $h_{1}$ was also studied in [2, 6]. For the next-to-leading order (NLO) $Q^{2}$ evolution, we need two-loop anomalous dimensions. So far two-loop anomalous dimension was calculated for $f_{1}\left[7\right.$, 8, 9, 10, 11, 12] and for $g_{1}[13$, 14. They have been used for the phenomenological parametrization of $f_{1}$ [15] and $g_{1}$ [16] to analyze accumulated experimental data.

The purpose of this paper is to calculate the two-loop anomalous dimension for $h_{1}$, following the method of $[7,8]$. We calculate the two-loop correction to the two-point Green function which imbeds the twist-2 operators for $h_{1}\left(x, Q^{2}\right)$. The calculation is done in the MS scheme in the dimensional regularization.

The construction of this paper is the following: In section 2, we briefly recall the twist-2 operators for the distribution $h_{1}$ and summarize the formal aspect of the next-to-leading order (NLO) effect in the $Q^{2}$-evolution of $h_{1}$. In section 3 , we describe the actual procedure of our calculation. Although the method is in parallel with that of [7, \&], presence of the free Lorentz index $\mu$ in the twist-2 operators for $h_{1}$ brings additional complication. We shall describe this peculiar feature for $h_{1}$ in great detail, but omit the details which are common to both $h_{1}$ and $f_{1}$. In section 4 , we present the results and discuss characteristic features of the NLO $Q^{2}$-evolution of $h_{1}\left(x, Q^{2}\right)$. Readers not interested in the technical details can directly proceed to this section. Section 5 is devoted to a brief summary. In Appendix A, we present the necessary integral formulas to express the anomalous dimension contribution from each diagram. In Appendix B, we give the contribution to the anomalous dimension from each two-loop diagram. 


\section{Twist-two operator for $h_{1}\left(x, Q^{2}\right)$ and its renormaliza- tion}

The transversity distribution $h_{1}\left(x, Q^{2}\right)$ of the nucleon is defined by the following relation [3]:

$$
\begin{aligned}
\int \frac{d \lambda}{2 \pi} e^{i \lambda x} & \left\langle P S\left|\bar{\psi}(0) \sigma_{\mu \nu} i \gamma_{5} \psi(\lambda n)\right|_{Q} \mid P S\right\rangle=2\left[h_{1}\left(x, Q^{2}\right)\left(S_{\perp \mu} p_{\nu}-S_{\perp \nu} p_{\mu}\right) / M\right. \\
& \left.+h_{L}\left(x, Q^{2}\right) M\left(p_{\mu} n_{\nu}-p_{\nu} n_{\mu}\right)(S \cdot n)+h_{3}\left(x, Q^{2}\right) M\left(S_{\perp \mu} n_{\nu}-S_{\perp \nu} n_{\mu}\right)\right]
\end{aligned}
$$

where $|P S\rangle$ is the nucleon (mass $M$ ) state specified by the four momentum $P$ and the spin vector $S$, and two light-like vectors $p$ and $n$ are introduced by the relation, $P^{\mu}=p^{\mu}+\frac{M^{2}}{2} n^{\mu}$ and $p n=1$. $S^{\mu}$ is decomposed as $S^{\mu}=(S \cdot n) p^{\mu}+(S \cdot p) n^{\mu}+S_{\perp}^{\mu}$. In (2.1), the gauge link operator to ensure the gauge invariance is suppressed. $h_{L}$ and $h_{3}$ are twist-3 and -4 distributions which are not in our interest here. Taylor expanding the both sides of (2.1) with respect to $\lambda$, one can relates the $n$-th moments of $h_{1, L}$ to the following local operator:

$$
\theta_{\mu \nu \mu_{1} \ldots \mu_{n}}=\mathcal{S}_{n} \bar{\psi} i \gamma_{5} \sigma_{\mu \nu} i D_{\mu_{1}} \ldots i D_{\mu_{n}} \psi
$$

where $\mathcal{S}_{n}$ denotes the symmetrization among $\nu, \mu_{1}, \cdots, \mu_{n}$, and the covariant derivative $D_{\mu}=$ $\partial_{\mu}-i g T^{a} A_{\mu}^{a}$ restores the gauge invariance. In particular, the $n$-th moment of $h_{1}$ is related to a tower of the twist-2 operators:

$$
\begin{aligned}
& \int_{-1}^{1} d x x^{n} h_{1}\left(x, Q^{2}\right)=a_{n}\left(Q^{2}\right) \\
& \left\langle P S\left|\bar{\theta}_{\mu \nu \mu_{1} \cdots \mu_{n}}(Q) \Delta^{\nu} \Delta^{\mu_{1}} \cdots \Delta^{\mu_{n}}\right| P S\right\rangle \\
& \quad=\frac{2}{M} a_{n}(Q)\left(S_{\mu} \hat{P}^{n+1}-P_{\mu} \hat{S} \hat{P}^{n}+\frac{2}{n+2} M^{2} \Delta_{\mu} \hat{S} \hat{P}^{n-1}\right),
\end{aligned}
$$

where $\bar{\theta}_{\mu \nu \mu_{1} \cdots \mu_{n}}$ is defined as the traceless part of $\theta_{\mu \nu \mu_{1} \cdots \mu_{n}}$ defined by the condition,

$$
g^{\mu \mu_{i}} \bar{\theta}_{\mu \nu \mu_{1} \ldots \mu_{n}}=g^{\nu \mu_{i}} \bar{\theta}_{\mu \nu \mu_{1} \ldots \mu_{n}}=g^{\mu_{i} \mu_{j}} \bar{\theta}_{\mu \nu \mu_{1} \ldots \mu_{n}}=0,
$$

and we introduced a null vector $\Delta^{\mu}\left(\Delta^{2}=0\right)$ which kills off the trace terms in $\bar{\theta}_{\mu \nu \mu_{1} \cdots \mu_{n}}$ as usual.

For simplicity we symbolically use the notation $O_{n}$ for $\bar{\theta}_{\mu \nu \mu_{1} \cdots \mu_{n}}$ in what follows in this section. The bare- $\left(O_{n}^{B}\right)$ and the renormalized- $\left(O_{n}(\mu)\right)$ composite operators are related by the renormalization constant $Z_{n}(\mu)$ for $O_{n}$ as

$$
O_{n}(\mu)=Z_{n}^{-1}(\mu) O_{n}^{B}
$$

The scale dependence of $O_{n}(\mu)$ is obtained by solving the renormalization group equation

$$
\mu \frac{d O_{n}(\mu)}{d \mu}+\gamma_{n}(g(\mu)) O_{n}(\mu)=0,
$$

where $\gamma_{n}(g(\mu))$ is the anomalous dimension for the operator $O_{n}(\mu)$ defined as

$$
\gamma_{n}(g(\mu))=\mu \frac{\partial}{\partial \mu} \ln Z_{n}(\mu) .
$$


This equation is solved to give

$$
O_{n}\left(Q^{2}\right)=O_{n}\left(\mu^{2}\right) \exp \left[-\int_{g\left(\mu^{2}\right)}^{g\left(Q^{2}\right)} d g \frac{\gamma_{n}(g)}{\beta(g)}\right] .
$$

Up to next-to-leading order, the anomalous dimension $\gamma_{n}(\mu)$ and the beta function $\beta(g)$ can be expanded as

$$
\begin{aligned}
\gamma_{n}(g) & =\frac{g^{2}}{16 \pi^{2}} \gamma_{n}^{(0)}+\frac{g^{4}}{\left(16 \pi^{2}\right)^{2}} \gamma_{n}^{(1)}+O\left(g^{6}\right), \\
\beta(g) & =-\frac{g^{3}}{16 \pi^{2}} \beta_{0}-\frac{g^{5}}{\left(16 \pi^{2}\right)^{2}} \beta_{1}+O\left(g^{7}\right),
\end{aligned}
$$

where the coefficients of the $\beta$-functions are well known; $\beta_{0}=11-(2 / 3) N_{f}, \beta_{1}=102-$ $(38 / 3) N_{f}$, with the number of quark flavor $N_{f}$. Inserting these equations into (2.9), one obtains the next-to-leading order evolution equation for the $n$-th moment of $h_{1}$ as

$$
\frac{\mathcal{M}_{n}\left[h_{1}\left(Q^{2}\right)\right]}{\mathcal{M}_{n}\left[h_{1}\left(\mu^{2}\right)\right]}=\left(\frac{g^{2}\left(Q^{2}\right)}{g^{2}\left(\mu^{2}\right)}\right)^{\frac{\gamma_{n}^{(0)}}{2 \beta_{0}}}\left(\frac{\beta_{0}+\beta_{1} g^{2}\left(Q^{2}\right) / 16 \pi^{2}}{\beta_{0}+\beta_{1} g^{2}\left(\mu^{2}\right) / 16 \pi^{2}}\right)^{\frac{1}{2}\left(\frac{\gamma_{n}^{(1)}}{\beta_{1}}-\frac{\gamma_{n}^{(0)}}{\beta_{0}}\right)},
$$

with

$$
\frac{g^{2}\left(Q^{2}\right)}{16 \pi^{2}}=\frac{1}{\beta_{0} \ln \left(Q^{2} / \Lambda^{2}\right)}\left[1-\frac{\beta_{1} \ln \ln \left(Q^{2} / \Lambda^{2}\right)}{\beta_{0}^{2} \ln \left(Q^{2} / \Lambda^{2}\right)}+\cdots\right],
$$

where we introduced the shorthand notation $\mathcal{M}_{n}\left[h_{1}(\mu)\right] \equiv \int_{-1}^{1} d x x^{n} h_{1}(x, \mu)$. This NLO effect in $h_{1}$ has to be combined with the NLO effect in the hard cross section in the parton level to give a prediction for a relevant physical cross section in the NLO level.

In the MS scheme of the dimensional regularization, one obtains $Z_{n}(\mu)$ as

$$
Z_{n}(\mu)=1+\sum_{k=1}^{\infty} \frac{X_{k}^{n}(g(\mu))}{\varepsilon^{k}},
$$

with $\varepsilon=(4-d) / 2$. Then the anomalous dimension is obtained as

$$
\gamma_{n}(g)=-2 g^{2} \frac{\partial X_{1}^{n}}{\partial g^{2}} .
$$

Therefore $\gamma_{n}^{(1)}$ in (2.10) is -4 times the coefficient of the $g^{4} /\left(16 \pi^{2}\right)^{2}$ term in $X_{1}^{n}(\mu)$. (See [7] for the detail.)

\section{Calculation of the two-loop anomalous dimension}

To obtain the renormalization constants for $\bar{\theta}^{\mu \nu \mu_{1} \cdots \mu_{n}}$ in (2.4), we calculate the two-loop correction to the two-point function for $\bar{\theta}$ with off-shell quark lines having the momentum $p$ $\left(p^{2}<0\right)$. The calculation of the two-loop Feynman diagram is rather involved, and we follow 
the procedure of [0], 8]. To discuss renormalization of $\bar{\theta}$, the presence of $i \gamma_{5}$ is irrelevant, since $i \gamma_{5} \sigma^{\mu \nu}=-1 / 2 \epsilon^{\mu \nu \kappa \rho} \sigma_{\kappa \rho}$. We shall henceforth omit $i \gamma_{5}$ for notational simplicity and consider the renormalization of the operator $O_{n}^{\mu \nu \mu_{1} \cdots \mu_{n}}$ defined by

$$
O_{n}^{\mu \nu \mu_{1} \cdots \mu_{n}}=\mathcal{S} \mathcal{T}_{n} \bar{\psi} \sigma^{\mu \nu} i D^{\mu_{1}} \cdots i D^{\mu_{n}} \psi
$$

where $\mathcal{S} \mathcal{T}_{n}$ denotes the symmetrization among $\nu, \mu_{1}, \cdots \mu_{n}$ and the traceless condition in (2.5). As usual, we contract $O_{n}^{\mu \nu \mu_{1} \cdots \mu_{n}}$ with a null vector $\Delta^{\mu}$ to kill off the trace terms, and consider the renormalization of $O_{n}^{\mu} \cdot \Delta \equiv O_{n}^{\mu \nu \mu_{1} \cdots \mu_{n}} \Delta_{\nu} \Delta_{\mu_{1}} \cdots \Delta_{\mu_{n}}$ in the following. The calculation is done in the Feynman gauge for the gluon propagator, but the results should be independent of the gauge.

We first need the Feynman rule for the basic vertices for the operator $O_{n}^{\mu} \cdot \Delta$ with 0,1 , 2 gluon lines shown in Fig.1:

$$
\begin{aligned}
& O_{n}^{(0) \mu} \cdot \Delta= \sigma^{\mu \nu} \Delta_{\nu} \hat{p}^{n} \\
& O_{n}^{(1) \mu} \cdot \Delta=g T^{a} \sigma^{\mu \nu} \Delta_{\nu} \sum_{j=1}^{n}{\hat{p^{\prime}}}^{j-1} \Delta^{\lambda} \hat{p}^{n-j} \\
& O_{n}^{(2) \mu} \cdot \Delta=g^{2} \sigma^{\mu \nu} \Delta_{\nu} {\left[\sum_{1 \leq j<l \leq n} \hat{p}^{j-1}\left(\hat{p}+\hat{k}^{\prime}\right)^{l-j-1} \hat{p}^{n-l} \Delta_{\lambda} \Delta_{\rho} T^{a} T^{b}\right.} \\
&\left.+\sum_{1 \leq j<l \leq n} \hat{p}^{j-1}(\hat{p}+\hat{k})^{l-j-1} \hat{p}^{n-l} \Delta_{\lambda} \Delta_{\rho} T^{b} T^{a}\right]
\end{aligned}
$$

where we introduced the notation $\hat{p} \equiv p \cdot \Delta$ for a four vector $p$. If one replaces $\sigma^{\mu \nu} \Delta_{\nu}$ by $\Delta$ in (3.2)-(3.4), one obtains the Feynman rule for the same basic vertices for $f_{1}$, which are used in [7]. Relevant two-loop diagrams are shown in Figs. 2, 3 and 4. Diagrams shown in Fig. 3 are only for the flavor-singlet distribution, but they are identically zero for $h_{1}$ owing to its chiral-odd nature. Those in Fig. 4 are the self energy corrections and are given in [7].

Among the contribution from each diagram in Fig. 2, we can show that the figs. (d), $(\mathrm{g}),(\mathrm{h}),(\mathrm{l}),(\mathrm{m}),(\mathrm{q})$ and $(\mathrm{r})$ give the same anomalous dimension to $h_{1}$ as the $f_{1}$ distribution calculated in [7]. To see this, we note the relation

$$
\sigma^{\mu \nu} \Delta_{\nu}=i \gamma^{\mu} \Delta-i \Delta^{\mu}=-i \Delta \gamma^{\mu}+i \Delta^{\mu}
$$

The term proportional to $\Delta^{\mu}$ is the trace term, and thus we can drop them and use either $i \gamma^{\mu} \Delta$ or $-i \Delta \gamma^{\mu}$ instead of $\sigma^{\mu \nu} \Delta_{\nu}$ in the actual calculation. We shall henceforth use the three forms for the vertex factor, $\sigma^{\mu \nu} \Delta_{\nu}, \gamma^{\mu} \Delta,-\Delta \gamma^{\mu}$, interchangeably (we also drop factor $i$ for simplicity) without mentioning explicitly. Therefore the difference in the Feynman rule between $h_{1}$ and $f_{1}$ is merely the presence of $i \gamma^{\mu}$ next to $\Delta$. The loop corrections in the above diagrams are located in the right or left half of the diagram and hence the presence of $\gamma^{\mu}$ does not bring any change in the calculation of the renormalization constants compared with the $f_{1}$ case. We can thus take the results from [7] for these diagrams.

It is also easy to confirm that the diagrams (a), (b) and (c) become finite due to the form of the vertex, and hence no contribution to the anomalous dimension unlike the case for $f_{1}$. Diagram (p) has no contribution as in the case of $f_{1}$. Calculation of (e) and (f) is 
also standard. One gets contribution from these two diagrams which are independent of $n$ unlike $f_{1}$ case.

Rather heavy calculation is required for the diagrams (i), (j), (k), (n) and (o). Compared with the renormalization of $f_{1}$, additional complication is caused by the presence of the free Lorentz index $\mu$ in $O_{n}^{\mu} \cdot \Delta$. To explain our strategy of the calculation, we take the diagram Fig. 2 (i) as an example. We assign the momentum as shown in Fig. 5. Application of the Feynman rule results in the following contribution from the diagram.

$$
\begin{gathered}
F^{(i)}=g^{4} \frac{1}{2 N_{c}} C_{F} \int D k_{1} \int D k_{2} \frac{\hat{k}_{1}^{n} N\left(k_{1}, k_{2}\right)}{D\left(k_{1}, k_{2}\right)}, \\
D\left(k_{1}, k_{2}\right)=k_{1}^{4} k_{2}^{2}\left(k_{1}-p\right)^{2}\left(k_{2}-p\right)^{2}\left(k_{1}-k_{2}\right)^{2}, \\
N\left(k_{1}, k_{2}\right)=\gamma_{\lambda} \not k_{1} \sigma^{\mu \nu} \Delta_{\nu} \not k_{1} \gamma_{\rho}\left(\not k_{1}-\not k_{2}\right) \gamma^{\lambda}\left(\not p-\not k_{2}\right) \gamma^{\rho},
\end{gathered}
$$

where

$$
\int D k_{1} \equiv \int \frac{d^{d} k_{1}}{(2 \pi)^{d}} .
$$

Our task is to extract from (3.6) the $1 / \varepsilon$ pole terms proportional to $\sigma^{\mu \nu} \Delta_{\nu} \hat{p}^{n}$. To this end we first contract the index $\rho$ in the numerator (3.8), noting the properties of the $\gamma$ matrix in $d=4-2 \varepsilon$ dimension. This gives

$$
\begin{aligned}
& N\left(k_{1}, k_{2}\right)=-2(1+\varepsilon) \gamma_{\lambda} \not k_{1} \sigma^{\mu \nu} \Delta_{\nu} \not \not k_{1}\left(\not p-\not k_{2}\right) \gamma^{\lambda}\left(\not k_{1}-\not k_{2}\right) \\
& +4 \varepsilon\left(\not k_{1}-\not \not_{2}\right) \not \not_{1} \sigma^{\mu \nu} \Delta_{\nu} \not k_{1}\left(\not p-\not k_{2}\right) \\
& +4 \varepsilon\left(\not p-\not \not_{2}\right) \not k_{1} \sigma^{\mu \nu} \Delta_{\nu} \not \not_{1}\left(\not k_{1}-\not \not_{2}\right) \\
& -4 \varepsilon\left(k_{1}-k_{2}\right) \cdot\left(p-k_{2}\right) \gamma_{\lambda} \not k_{1} \sigma^{\mu \nu} \Delta_{\nu} \not k_{1} \gamma^{\lambda} \text {. }
\end{aligned}
$$

Noting $\gamma_{\lambda} \not k_{1} \sigma^{\mu \nu} \Delta_{\nu} \not k_{1} \gamma^{\lambda}=-2 \varepsilon \not k_{1} \sigma^{\mu \nu} \Delta_{\nu} \not k_{1}$, the last term of (3.10) becomes $O\left(\varepsilon^{2}\right)$ and cannot produce $1 / \varepsilon$-poles so that we can drop it. Using the same relation in the first term, one gets up to $O(\varepsilon)$

$$
\begin{aligned}
N\left(k_{1}, k_{2}\right)= & -4\left(\not p-\not k_{2}\right) \not k_{1} \sigma^{\mu \nu} \Delta_{\nu} \not \not_{1}\left(\not \not_{1}-\not \not_{2}\right)-4 \varepsilon \not k_{1} \sigma^{\mu \nu} \Delta_{\nu} \not k_{1}\left(\not p-\not k_{2}\right)\left(\not k_{1}-\not k_{2}\right) \\
& +4 \varepsilon\left(\not \not_{1}-\not k_{2}\right) \not k_{1} \sigma^{\mu \nu} \Delta_{\nu} \not k_{1}\left(\not p-\not k_{2}\right) .
\end{aligned}
$$

In the first and the third terms in (3.11), we can discard the terms which have $\not p$ at the leftmost or the rightmost places, since those terms are either ultraviolet finite from power counting or only cause trace terms which are proportional to $\Delta^{\mu}$. In fact, for example, one can write

$$
\begin{gathered}
\int D k_{1} \int D k_{2} \frac{{\hat{k_{1}}}^{n} \not p \not k_{1} \gamma^{\mu} \Delta \not k_{1} \not k_{2}}{D\left(k_{1}, k_{2}\right)}=A \not p \gamma^{\mu} \hat{p}^{n+1}\left(p^{2}\right)^{-(1+2 \varepsilon)}+B p^{\mu} \not p \Delta \hat{p}^{n}\left(p^{2}\right)^{-(1+2 \varepsilon)} \\
+B^{\prime} p^{\mu} \hat{p}^{n+1}\left(p^{2}\right)^{-(1+2 \varepsilon)}+C \Delta^{\mu} \not p \Delta \hat{p}^{n-1}\left(p^{2}\right)^{-2 \varepsilon}+C^{\prime} \Delta^{\mu} \hat{p}^{n}\left(p^{2}\right)^{-2 \varepsilon}
\end{gathered}
$$


where $A, B$ and $B^{\prime}$ terms are finite, and $C$ and $C^{\prime}$ terms are the trace terms. Dropping these terms, one can rewrite (3.11) as follows:

$$
\begin{aligned}
N\left(k_{1}, k_{2}\right)= & -4 k_{1}^{2} \not k_{2} \not k_{1} \Delta \gamma^{\mu}-4 \varepsilon k_{1}^{2} \gamma^{\mu} \Delta \not k_{1} \not k_{2}-4(1-\varepsilon) \not k_{2} \not k_{1} \gamma^{\mu} \Delta \not k_{1} \not k_{2} \\
& +4 \varepsilon\left(2 k_{1} \cdot k_{2}-2 p \cdot k_{1}+2 p \cdot k_{2}-k_{2}^{2}\right) \not k_{1} \gamma^{\mu} \Delta \not k_{1}-4 \varepsilon k_{1}^{2} \not k_{1} \gamma^{\mu} \Delta \not k_{2} .
\end{aligned}
$$

The integration of the first and the second terms in (3.13) can be handled as follows. One can write

$$
\int D k_{1} \int D k_{2} \frac{k_{1}^{2} \hat{k}_{1}^{n} \not k_{2} \not k_{1} \Delta \gamma^{\mu}}{D\left(k_{1}, k_{2}\right)}=A \Delta \gamma^{\mu}+(\text { finite as } \varepsilon \rightarrow 0) \text {, }
$$

where $A$ can contain a pole at $\varepsilon=0$. The $A$ term can be projected out by multiplying $p^{\mu}$ to both sides of (3.14) and taking trace of both sides. This is equivalent to replace in the numerator of (3.14) as

$$
\not k_{2} \not k_{1} \Delta \gamma^{\mu} \rightarrow \frac{1}{\hat{p}}\left(\hat{p} k_{1} \cdot k_{2}+\hat{k}_{1} p \cdot k_{2}-\hat{k}_{2} p \cdot k_{1}\right) \Delta \gamma^{\mu}
$$

Similarly to (3.15), we can replace in the second term of (3.13) as

$$
\gamma^{\mu} \Delta \not k_{1} \not k_{2} \rightarrow \frac{1}{\hat{p}}\left(\hat{p} k_{1} \cdot k_{2}+\hat{k}_{1} p \cdot k_{2}-\hat{k}_{2} p \cdot k_{1}\right) \gamma^{\mu} \Delta
$$

After the replacement (3.15) in (3.14), the integration can be easily done by the same method as [7]: For example, $k_{1} \cdot k_{2}$ in the first term of (3.15) is replaced by $1 / 2\left(k_{1}^{2}+k_{2}^{2}-\left(k_{1}-k_{2}\right)^{2}\right)$. Then each of these three terms combined with the $k_{1}^{2}$ factor in (3.14) cancel some of the factors in the denominator $D\left(k_{1}, k_{2}\right)$ in (3.7), leaving the $O\left(k_{1,2}^{8}\right)$ factor in the denominator. After this cancellation, one needs only one Feynman parameter for each integration $\left(\int D k_{1}\right.$ and $\int D k_{2}$ ) and thus one can easily carry out the integration.

As is shown in this example, our strategy of the calculation is to devise a suitable replacement for each term in the numerator to project out the pole terms proportional to $\gamma^{\mu} \Delta$ or $\Delta \gamma^{\mu}$, ignoring the trace term $\left(\sim \Delta^{\mu}\right)$. After this projection, it turns out that we can express the final formula for the pole contributions of the two-loop diagrams in terms of the integral formula $I_{1}$ to $I_{14}$ listed in the appendix of [8] and two new integrals $I_{15}$ and $I_{16}$ shown in the Appendix.

As was stated in (2.15), simple pole terms $(\sim 1 / \varepsilon)$ from each two-loop Feynman diagram give rise to the anomalous dimension. There are two origins for the simple poles: (i) $O\left(\varepsilon^{0}\right)$ contribution in the numerator combined with the $1 / \varepsilon$ singularity from the integral. (ii) $O(\varepsilon)$ contribution in the numerator combined with the $1 / \varepsilon^{2}$ singularity from the integral. For the type-(i) singularity, one can subtract the counter terms after transforming each twoloop integral into the form of $I_{1} \sim I_{16}$. Therefore, in the integral formulas given in [8] and Appendix A, the counter terms for the simple pole terms are already subtracted. To subtract the counter terms for the type-(ii) $1 / \varepsilon$-pole, we have to come back to the original diagram [7]. In Fig. 6, we schematically showed the counter term contribution for the diagram (i) in Fig. 2. In Fig. 6(a), the counter-term corresponds to the renormalization of the operator in one-loop (Fig. 6(b)), but the numerator of the diagram in Fig. 6(a) has no $\varepsilon$. In Fig. 6(c) 
the counter-term comes from the one-loop contribution to the vertex correction (Fig. 6(d)). This time the numerator of the diagram in Fig. 6(c) becomes $O\left(\varepsilon^{2}\right)$, hence no counter-term contribution. To summarize: we only have to subtract the counter-terms for the simple poles in $I_{1} \sim I_{16}$ for the diagram (i). This is also true for other diagrams in Fig. 2.

The integration of the third term in (3.13) is rather demanding. We repeatedly apply the anti-commutation relation to move the leftmost $\not k_{2}$ untill it merges the righmost $\not k_{2}$ to form $\not k_{2} \not k_{2}=k_{2}^{2}$. One then obtains

$$
\begin{aligned}
& \not k_{2} \not k_{1} \gamma^{\mu} \Delta \not k_{1} \not k_{2}=2 k_{1} \cdot k_{2} \gamma^{\mu} \Delta \not k_{1} \not k_{2}-4 \hat{k}_{1} k_{2}^{\mu} \not k_{1} \not k_{2}+2 k_{1}^{2} k_{2}^{\mu} \Delta \not k_{2} \\
& +4 \hat{k}_{2} k_{1}^{\mu} \not k_{1} \not k_{2}-2 \hat{k}_{2} k_{1}^{2} \gamma^{\mu} \not k_{2}-2 k_{1} \cdot k_{2} \not k_{1} \gamma^{\mu} \Delta \not k_{2}+k_{2}^{2} \not k_{1} \gamma^{\mu} \Delta \not k_{1} .
\end{aligned}
$$

The first term of (3.17) can be handled in the same way as (3.16).

The fifth term of (3.17) is the easiest to deal with. One only has to make the replacement

$$
\gamma^{\mu} \not k_{2} \rightarrow \frac{p \cdot k_{2}}{\hat{p}} \gamma^{\mu} \Delta
$$

by the same argument as in (3.14).

The third term of (3.17) can be treated as follows. To meet with more general situation we replace $k_{2}^{\mu}$ by $k_{1}^{\mu}$ ( $k_{1}^{\mu}$ can be put equal to $k_{1}^{\mu}$ or $k_{2}^{\mu}$ in the following formula.) From power counting, one can write

$$
\int D k_{1} \int D k_{2} \frac{\hat{k}_{1}^{n} k_{1}^{2} k_{1}^{\mu} \Delta \not k_{2}}{D\left(k_{1}, k_{2}\right)}=A \Delta \gamma^{\mu}+B \Delta^{\mu} \Delta \not p+(\text { finite as } \varepsilon \rightarrow 0),
$$

where $A$ and $B$ can contain poles at $\varepsilon=0$. To project out the $A$-term, we multiply $\not p \gamma^{\mu}$ from the left and take the trace. To $O(\varepsilon)$, this is equivalent to the replacement,

$$
k_{1}^{\mu} \Delta \not k_{2} \rightarrow \frac{-1}{2 \hat{p}}(1+\varepsilon)\left(\hat{k}_{1} p \cdot k_{2}+\hat{k}_{2} p \cdot k_{1}-\hat{p} k_{1} \cdot k_{2}\right) \Delta \gamma^{\mu}
$$

in the numerator of (3.19). Similarly to (3.20), we often meet the following replacement in the calculation:

$$
k_{1}^{\mu} \not k_{2} \Delta \rightarrow \frac{-1}{2 \hat{p}}(1+\varepsilon)\left(\hat{k}_{1} p \cdot k_{2}+\hat{k}_{2} p \cdot k_{1}-\hat{p} k_{1} \cdot k_{2}\right) \gamma^{\mu} \Delta
$$

We can deal with the second and fourth term of (3.17) as follows. Without loss of generality one can write $\left(q^{\mu}=k_{1}^{\mu}\right.$ or $\left.k_{2}^{\mu}\right)$

$$
\int D k_{1} \int D k_{2} \frac{\hat{k}_{1}^{n+1} q^{\mu} \not k_{1} \not k_{2}}{D\left(k_{1}, k_{2}\right)}=A \gamma^{\mu} \Delta+B \Delta^{\mu}+C \Delta^{\mu} \not p \Delta+(\text { finite as } \varepsilon \rightarrow 0) \text {, }
$$

where $A, B$ and $C$ can contain poles at $\varepsilon=0$, and we can ignore finite contribution. To project out the $A$-term, we combine two operations. (i) Multiply $p^{\mu} \Delta \not p$ from the right and take trace. (ii) Multiply $\gamma^{\mu} \not p \hat{p}$ from the right and take trace. Taking the difference between 
these two results, it turns out that the projection of the $A$-term in (3.22) is equivalent to the following replacement in the integrand:

$$
q^{\mu} \not k_{1} \not k_{2} \rightarrow \frac{1}{2 \hat{p}^{2}}(1+\varepsilon)\left\{\hat{p}\left(p \cdot k_{2} q \cdot k_{1}-p \cdot k_{1} q \cdot k_{2}\right)-p \cdot q\left(\hat{k}_{1} p \cdot k_{2}-\hat{k}_{2} p \cdot k_{1}\right)\right\} \gamma^{\mu} \Delta .
$$

The sixth term in (3.17) can be rewritten as $\not k_{1} \gamma^{\mu} \Delta \not k_{2}=2 k_{1}^{\mu} \Delta \not k_{2}-2 \hat{k}_{1} \gamma^{\mu} \not k_{2}+\gamma^{\mu} \Delta \not k_{1} \not k_{2}$ and we can apply (3.20), (3.18), (3.16) for each term, and likewise for the seventh term in (3.17).

As an application of the above replacement rules, we prove a usefull lemma here.

Lemma: A term of the form $\varepsilon \not k \sigma^{\mu \nu} \Delta_{\nu} \not k^{\prime}$, where $k$ and $k^{\prime}$ are either $k_{1}$ or $k_{2}$, does not give rise $1 / \varepsilon$ pole.

Proof: We write $\varepsilon \not k \gamma^{\mu} \Delta \not k^{\prime}=2 \varepsilon k^{\mu} \Delta \not k^{\prime}-2 \varepsilon \hat{k} \gamma^{\mu} \not k^{\prime}+\varepsilon \gamma^{\mu} \Delta \not k k^{\prime}$, and apply (3.20), (3.18), (3.16) for each term. Then it vanishes, ignoring $O\left(\varepsilon^{2}\right)$ term. This completes the proof.

Applying this lemma, we can drop fourth and the fifth terms in (3.13), which completes transformation of all terms in (3.13) into the requested forms as mentioned before. By this lemma we can drop many of the terms in the calculation of the diagrams $(\mathrm{i}),(\mathrm{j})$ and $(\mathrm{k})$.

The obtained rules for the replacement (3.15), (3.16), (3.18), (3.20), (3.21), (3.23) give all the necessary formula to carry out the integration of the two-loop diagrams. Using the method described in this section, we obtained the anomalous dimension from all the diagrams in Fig. 2. They are given in Appendix B.

\section{Results and discussions}

Collecting all the contribution from each diagram in Figs. 2 and 4 (See Appendix B and appendices of [7, 9]), we obtained $\gamma_{n}^{(1)}$ for $h_{1}$ as,

$$
\begin{aligned}
\gamma_{n}^{h(1)}= & 4 C_{F}^{2}\left[S_{2}(n+1)-2 S_{1}(n+1)-\frac{1}{4}\right] \\
& +C_{F} C_{G}\left[-16 S_{1}(n+1) S_{2}(n+1)-\frac{58}{3} S_{2}(n+1)+\frac{572}{9} S_{1}(n+1)-\frac{20}{3}\right] \\
& -8\left(C_{F}^{2}-\frac{1}{2} C_{F} C_{G}\right)\left[4 S_{1}(n+1)\left\{S_{2}^{\prime}\left(\frac{n+1}{2}\right)-S_{2}(n+1)-\frac{1}{4}\right\}-8 \widetilde{S}(n+1)\right. \\
& \left.+S_{3}^{\prime}\left(\frac{n+1}{2}\right)-\frac{5}{2} S_{2}(n+1)+\frac{\left(1+(-1)^{n}\right)}{(n+1)(n+2)}+\frac{1}{4}\right] \\
& +\frac{32}{9} C_{F} T_{R}\left[3 S_{2}(n+1)-5 S_{1}(n+1)+\frac{3}{8}\right]
\end{aligned}
$$

where

$$
\begin{aligned}
S_{k}(n) & =\sum_{j=1}^{n} \frac{1}{j^{k}}, \\
S_{k}^{\prime}\left(\frac{n}{2}\right) & =\frac{1+(-1)^{n}}{2} S_{k}\left(\frac{n}{2}\right)+\frac{1-(-1)^{n}}{2} S_{k}\left(\frac{n-1}{2}\right),
\end{aligned}
$$




$$
\widetilde{S}(n)=\sum_{j=1}^{n} \frac{(-1)^{j}}{j^{2}} S_{1}(j) .
$$

(Note that $2 / 3 S_{1}(n)$ in the seccond line of (A.8) in [9] should read $2 / 3 S_{3}(n)$.) This final formula for $h_{1}\left(x, Q^{2}\right)$ is very complicated as in the case of $f_{1}\left(x, Q^{2}\right)$ and is subject to numerical analysis to study its $Q^{2}$-evolution. In table 1 , we present the actual numbers of $\gamma_{n}^{h(1)}\left(\gamma_{n}^{(1)}\right.$ for $h_{1}$ ) for $n=0, \ldots, 20$. This table can be compared with Table 2 of [7], which presents $\gamma_{n}^{f(1)}\left(\gamma_{n}^{(1)}\right.$ for $\left.f_{1}\right)$. (Note that $n$ in [7] corresponds to our $n-1$.) We also plotted $\gamma_{n}^{h, f(1)}$ for $N_{f}=3$, 5 in Fig. 7 to compare their behavior. From Fig. 7, one sees clearly that at small $n \gamma_{n}^{h(1)}$ is significantly larger than $\gamma_{n}^{f(1)}$ but approaches very quickly to $\gamma_{n}^{f(1)}$, keeping the condition $\gamma_{n}^{h(1)}>\gamma_{n}^{f(1)}$. This feature is the same as the one-loop anomalous dimensions $\gamma_{n}^{h, f(0)}$ which reads

$$
\begin{aligned}
\gamma_{n}^{f(0)} & =2 C_{F}\left(1-\frac{2}{(n+1)(n+2)}+4 \sum_{j=2}^{n+1} \frac{1}{j}\right), \\
\gamma_{n}^{h(0)} & =2 C_{F}\left(1+4 \sum_{j=2}^{n+1} \frac{1}{j}\right),
\end{aligned}
$$

and hence $\gamma_{n}^{h(0)}>\gamma_{n}^{f(0)}$ for all $n$. Actually this tendency is even stronger for the two-loop case. We note that for $n=0$ the anomalous dimension for $f_{1}$ is zero in all orders because of the Ward identity for the vector current. On the other hand, $h_{1}$ projects onto the tensor operator $\bar{\psi} \sigma^{\mu \nu} \psi$ for $n=0$, for which there is no conservation law. Hence $\gamma_{0}^{h(0,1)} \neq 0$. This can be taken as a good reason for $\gamma_{n}^{h(0,1)}>\gamma_{n}^{f(0,1)}$ especially at small $n$. From the difference between $\gamma_{n}^{f(1)}$ and $\gamma_{n}^{h(1)}$ at small $n$, we expect that the NLO effect leads to larger difference in the $Q^{2}$-evolution in the small- $x$ region.

At large $n, \gamma_{n}^{h, f(1)}$ receives dominant contributions from diagrams (d), (g), (h), (m), (q), (r) in Fig. 2 which give the same anomalous dimension both for $h_{1}$ and $f_{1}$, and sub-dominant contribution from (n), (l), (f), (e) and the self energy corrections shown in Fig. 4. All other diagrams in Fig. 2 gives minor corrections, although they are very important at small $n$. As is discussed in [9], $\gamma_{n}^{f(1)}$ behaves as $\sim \ln (n)$ at large $n$ with the cancellation among $\sim \ln ^{2} n$ and $\sim \ln ^{3} n$ terms which arise from some of the diagrams. Equation (4.1) and quick merging between $\gamma_{n}^{h(1)}$ and $\gamma_{n}^{f(1)}$ at large $n(n \geq 10)$ with the relation $\gamma_{n}^{h(1)}>\gamma_{n}^{f(1)}$ for all $n$ clearly shows $\gamma_{n}^{h(1)} \sim \ln (n)$, which also supports the correctness of our calculation.

The relevant quantities for the $Q^{2}$-evolution of the moments are $\gamma_{n}^{(0)} / 2 \beta_{0}$ and $\gamma_{n}^{(1)} / 2 \beta_{1}$ as is seen from (2.12). In Fig. 8(a), we plotted these quantities for $f_{1}$ and $h_{1}$ with $N_{f}=3$. Since the NLO effect is determined by their difference (see (2.12)), we plotted $\gamma_{n}^{f, h(1)} / 2 \beta_{1}-$ $\gamma_{n}^{f, h(0)} / 2 \beta_{0}$ in Fig. 8(b) for $N_{f}=3$ and 5 cases. From Fig. 8(b), one expects that at small $n$ the NLO effect in the $Q^{2}$ evolution is quite different between $\mathcal{M}_{n}\left[h_{1}\left(Q^{2}\right)\right]$ and $\mathcal{M}_{n}\left[f_{1}\left(Q^{2}\right)\right]$. Since $\gamma_{n}^{f(0,1)} \rightarrow 0$ as $n \rightarrow 0, \gamma_{n}^{f(1)} / 2 \beta_{1}-\gamma_{n}^{f(0)} / 2 \beta_{0}$ abruptly drops to zero as $n \rightarrow 0$. But this is not the case for $h_{1}$ : The difference between the black circles and squares in Fig. 8(a) shows a characteristic behavior as shown in Fig. 8(b).

As an example of the $Q^{2}$-evolution, we show in Fig. 9 (a) and (b) the $Q^{2}$-evolution of the tensor charge and the first moments, respectively, with the parameters $N_{f}=3$ and $\Lambda=0.232$ $\mathrm{GeV}$ in (2.13). (Here we are interested in the NLO effect in the anomalous dimension and 
the $\beta$-function, and thus we adopted the same value for the $\Lambda$-parameter in the LO and NLO evolution.) They are normalized at $Q^{2}=1 \mathrm{GeV}^{2}$. At $n=0$, only diagrams (e), (f), (i), (j), (k) survive. They give the anomalous dimension for the tensor charge as

$$
\begin{aligned}
\gamma_{0}^{h(1)} & =-19 C_{F}^{2}+\frac{257}{9} C_{F} C_{G}-\frac{52}{9} C_{F} T_{R} \\
& =\frac{724}{9}-\frac{104}{27} N_{f} .
\end{aligned}
$$

From Fig. 9(a), we can compare the LO and NLO $Q^{2}$-evolution of the tensor charge of the nucleon. One sees that the NLO effect is sizable as is expected from Fig. 8 (b). In Fig. 9(b), we plotted the LO and NLO $Q^{2}$-evolution both for $\mathcal{M}_{1}\left[f_{1}\left(Q^{2}\right)\right]$ and $\mathcal{M}_{1}\left[h_{1}\left(Q^{2}\right)\right]$. Although the NLO effect in the anomalous dimension (the second factor in the right hand side of (2.12)) makes this ratio smaller, the NLO effect in the coupling constant (the first factor in the right hand side of (2.12) ) completely cancels this effect. For $f_{1}$, the latter effect is actually bigger than the former effect. As was stated in section 2, the NLO effect in the distribution function has to be combined with the NLO effect in the hard cross section in the parton level to give a prediction for a physical quantity. We will pursue this issue in a future publication.

Table 1 The coefficients of the two-loop anomalous dimension $\gamma_{n}^{(1)}$ for $h_{1}$ with the number of quark flavors $N_{f}=3,4,5$.

\begin{tabular}{|c|r|r|r|}
\hline \multirow{2}{*}{$n$} & \multicolumn{3}{|c|}{$\gamma_{n}^{(1)}$} \\
\cline { 2 - 4 } & $N_{f}=3$ & \multicolumn{1}{|c|}{4} & \multicolumn{1}{c|}{5} \\
\hline 0 & 68.89 & 65.04 & 61.19 \\
1 & 100.00 & 92.00 & 84.00 \\
2 & 123.08 & 111.92 & 100.76 \\
3 & 140.51 & 126.83 & 113.15 \\
4 & 155.36 & 139.60 & 123.83 \\
5 & 167.87 & 150.33 & 132.79 \\
6 & 179.02 & 159.93 & 140.84 \\
7 & 188.84 & 168.38 & 147.92 \\
8 & 197.81 & 176.12 & 154.43 \\
9 & 205.91 & 183.11 & 160.31 \\
10 & 213.43 & 189.61 & 165.79 \\
11 & 220.34 & 195.58 & 170.82 \\
12 & 226.82 & 201.19 & 175.56 \\
13 & 232.84 & 206.40 & 179.96 \\
14 & 238.54 & 211.34 & 184.14 \\
15 & 243.88 & 215.97 & 188.06 \\
16 & 248.96 & 220.38 & 191.79 \\
17 & 253.77 & 224.55 & 195.32 \\
18 & 258.36 & 228.53 & 198.71 \\
19 & 262.72 & 232.32 & 201.92 \\
20 & 266.91 & 235.96 & 205.01 \\
\hline
\end{tabular}




\section{Summary}

In this paper, we have carried out the calculation of the two-loop anomalous dimension for the transversity distribution $h_{1}\left(x, Q^{2}\right)$. The calculation was done in the MS scheme of the dimensional regularization. This completes the calculation of the anomalous dimensions for all the twist-2 distributions of the nucleon in the NLO level. We found $\gamma_{n}^{h(1)}$ is significantly larger than $\gamma_{n}^{f(1)}$ at small $n$, but approaches very quickly to $\gamma_{n}^{f(1)}$ at large $n$, keeping the condition $\gamma_{n}^{h(1)}>\gamma_{n}^{f(1)}$. This means that the NLO effect for $h_{1}$ is quite different from that for $f_{1}$ in the small $x$-region. As an example, we have compared the LO and the NLO $Q^{2}$ evolution of the tensor charge and the first moment of $f_{1}$ and $h_{1}$. We hope the peculiar feature of the $Q^{2}$-evolution of $h_{1}$ studied in this paper will be measured in the ongoing and planned experiments in the near future.

\section{Note added:}

After submission of this work for publication, there appeared a preprint [17] which calculated the NLO splitting function for $h_{1}$ by a different method. Our whole result agrees with [17]. After submission, we also learned that there appeared a preprint [18, which calculated the two-loop anomalous dimension for $h_{1}$ in the same formalism as ours, but presented the result in a very complicated non-final form. We further noticed that (1) their result for the diagrams Fig. 2 (i), (j), (k) disagrees with ours, (2) their result for Fig. 2 (n) and (o) agrees with our intermediate result but are not presented in a simple form. We believe something must be wrong in their calculation. 


\section{APPENDIX}

\section{A. Integral formula}

To write down the two-loop anomalous dimension for $h_{1}$, it turns out that we need two more integral formula, $I_{15}$ and $I_{16}$, in addition to $I_{1} \sim I_{14}$ defined in the appendix of [8]. As in the case of $I_{1} \sim I_{14}$, we define them by subtracting the counter term contribution for the simple-pole term but not subtracting the counter-term for the double-pole term as explained in the text:

$$
\begin{aligned}
I_{15}(M, 0) \equiv & \int \frac{d^{D} k_{1}}{(2 \pi)^{D}} \frac{d^{D} k_{2}}{(2 \pi)^{D}} \frac{\hat{k}_{1}^{M} k_{1} \cdot k_{2}}{k_{1}^{4}\left(k_{1}-p\right)^{2}\left(k_{2}-p\right)^{2}\left(k_{1}-k_{2}\right)^{2}} \\
I_{15}(M, 0)= & \frac{\hat{p}^{M}}{\left(16 \pi^{2}\right)^{2}} \frac{3}{4(M+1)}\left[-\frac{1}{2 \varepsilon^{2}}+\frac{1}{2 \varepsilon}\left\{\frac{1}{3 M}+\frac{1}{M+1}-2\right\}\right], \quad(M \geq 1) \\
I_{15}(0,0)= & \frac{-1}{4\left(16 \pi^{2}\right)^{2}}\left(\frac{1}{\varepsilon^{2}}+\frac{1}{\varepsilon}\right) \\
I_{16}(M, N) \equiv & \int \frac{d^{D} k_{1}}{(2 \pi)^{D}} \frac{d^{D} k_{2}}{(2 \pi)^{D}} \frac{\hat{k}_{1}^{M} \hat{k}_{2}^{N} k_{1} \cdot p}{k_{1}^{4}\left(k_{2}-p\right)^{2}\left(k_{1}-k_{2}\right)^{2}\left(k_{1}-k_{2}+p\right)^{2}} \\
I_{16}(M, N)= & \frac{\hat{p}^{M+N}}{2\left(16 \pi^{2}\right)^{2}} \frac{M ! N !}{(M+N) !} \sum_{k=0}^{N} \frac{(k+1) !(M+N-k-1) !}{(N-k) !(M+k+1) !}\left[-\frac{1}{2 \varepsilon^{2}}\right. \\
& \left.+\frac{1}{2 \varepsilon}\left\{S_{1}(M+N-k-1)+S_{1}(M+k+1)-2 S_{1}(M+N+1)\right\}\right] \\
I_{16}(0, N)= & \frac{-\hat{p}^{N}}{(4 \pi)^{4}} \frac{N}{4(N+1)}\left[\frac{1}{\varepsilon^{2}}+\frac{1}{\varepsilon} \frac{1}{N} \sum_{k=0}^{N-1}\left(2 S_{1}(N+1)\right.\right. \\
& \left.\left.-2 S_{1}(k+1)+S_{1}(k)-S_{N-k-1}-\frac{k+1}{k+2}\right)\right], \quad(N \geq 1) \\
I_{16}(0,0)= & 0,
\end{aligned}
$$

where we discarded the finite contributions. We also remind the readers that our $\varepsilon$ is $\varepsilon / 2$ in [7, 8].

Although the expression for $I_{1} \sim I_{14}$ are given in [8], some of them contain misprints. Furthermore, when we calculate the anomalous dimension for the tensor charge, we need to prepare some of $I_{1} \sim I_{14}$ separately as in the case of $I_{15,16}$. In the following, we present such integral formulas.

$$
\begin{aligned}
I_{3}(0, N) & =\frac{\hat{p}^{N}}{2\left(16 \pi^{2}\right)^{2}} \frac{1}{N+1}\left[\frac{1}{\varepsilon^{2}}-\frac{1}{\varepsilon} S_{1}(N+1)\right], \quad(N \geq 0) \\
I_{6}(M, 0) & \equiv \int \frac{d^{D} k_{1}}{(2 \pi)^{D}} \frac{d^{D} k_{2}}{(2 \pi)^{D}} \frac{\hat{k}_{1}^{M} k_{2} \cdot p}{k_{1}^{4}\left(k_{1}-p\right)^{2}\left(k_{2}-p\right)^{2}\left(k_{1}-k_{2}\right)^{2}}
\end{aligned}
$$




$$
\begin{aligned}
= & \frac{\hat{p}^{M}}{4\left(16 \pi^{2}\right)^{2}} \frac{1}{M+1}\left[-\frac{1}{2 \varepsilon^{2}}+\frac{1}{\varepsilon}\left\{\frac{1}{2(M+1)}+\frac{1}{2 M}-1\right\}\right], \quad(M \geq 1)(A \\
I_{8}(M, N) \equiv & \int \frac{d^{D} k_{1}}{(2 \pi)^{D}} \frac{d^{D} k_{2}}{(2 \pi)^{D}} \frac{\hat{k}_{1}^{M} \hat{k}_{2}^{N} k_{1} \cdot p}{k_{1}^{4} k_{2}^{2}\left(k_{2}-p\right)^{2}\left(k_{1}-k_{2}\right)^{2}} \\
I_{8}(M, N)= & \frac{\hat{p}^{M+N}}{4\left(16 \pi^{2}\right)^{2}} \frac{1}{(M+1)(M+N)}\left[-\frac{1}{\varepsilon^{2}}+\frac{1}{\varepsilon}\left\{S_{1}(M+N)-S_{1}(M)\right.\right. \\
& \left.\left.+\frac{1}{M+N+1}-\frac{1}{M}-\frac{2}{M+1}\right\}\right], \quad(M \geq 1, N \geq 0) \\
I_{8}(0, N)= & \frac{-\hat{p}^{N}}{4\left(16 \pi^{2}\right)^{2}} \frac{1}{N+1} \frac{1}{\varepsilon}, \quad(N \geq 0) \\
I_{11}(M, N) \equiv & \int \frac{d^{D} k_{1}}{(2 \pi)^{D}} \frac{d^{D} k_{2}}{(2 \pi)^{D}} \frac{\left(\hat{k}_{1}^{M} \hat{k}_{2}^{N}\right.}{k_{2}^{2}\left(k_{2}-p\right)^{2}\left(k_{1}-k_{2}\right)^{2}\left(k_{1}-k_{2}+p\right)^{2}} \\
& \frac{-\hat{p}^{M+N}}{\left(16 \pi^{2}\right)^{2}} \frac{1}{\varepsilon^{2}} \frac{1}{M+N+2}\left[\frac{1}{M+1}+\frac{(-1)^{M} M ! N !}{(M+N+1) !}\right]
\end{aligned}
$$

The summation in $I_{1}$ and $I_{13}$ should start from $J=0$ in (A.2) and (A.14) of [8].

All the other integral formula for $I_{1} \sim I_{14}$ which are necessary in our calculation but not presented explicitly in the above can be taken from Appendix of [8].

\section{B. Anomalous dimension from each diagram}

In this appendix, we present the contribution to $\gamma_{n}^{(1)}$ for $h_{1}$ from each Feynman diagram shown in Fig. 2, for which we use the notation $\gamma_{n}^{(a)}, \gamma_{n}^{(b)}$ etc with obvious definition. We also use the symbols $I_{1}^{D . P .}$ and $I_{1}^{S . P .}$ etc to designate, respectively, the coefficients of the $1 /\left(16 \pi^{2}\right)^{2} \varepsilon^{2}$ (double pole) term and the $1 /\left(16 \pi^{2}\right)^{2} \varepsilon$ (simple pole) term of the integrals $I_{1}$ through $I_{16}$ defined in the appendix of [8] and the appendix A above.

As noted in the text, $\gamma_{n}^{(a, b, c, p)}=0$, and $\gamma_{n}^{(d, g, h, l, m, q, r)}$ are the same as those for $f_{1}$ calculated in [7]. Other results are as follows: $\left(C_{F}=\left(N_{c}^{2}-1\right) / 2 N_{c}, C_{G}=N_{c}, T_{R}=N_{f} / 2\right.$ for the color group $\left.S U\left(N_{c}\right)\right)$ :

$$
\begin{gathered}
\gamma_{n}^{(e)}=-\frac{16}{9} C_{F} T_{R} . \\
\gamma_{n}^{(f)}=\frac{32}{9} C_{F} C_{G} .
\end{gathered}
$$

Following the calculational procedure described in section 3, we obtained very cumbersome results for $\gamma_{n}^{(i, j, k, n, o)}$ in terms of the integral formulas, $I_{1} \sim I_{16}$. It turned out, however, we can further simplify those results as was done in [9] for $f_{1}$. In the following, we present the simplified forms together with the expression with $I_{1} \sim I_{16}$. Although we had to calculate 
the integral formulas for $\gamma_{n=0}^{h(1)}$ separately from those for $\gamma_{n \geq 1}^{h(1)}$, the final results for (i), (j), (k) can be written in a very simple form for all $n$, which supports the correctness of the calculation.

$$
\begin{aligned}
& \gamma_{n}^{(i)}=16\left(C_{F}^{2}-\frac{1}{2} C_{F} C_{G}\right) \hat{p}^{-n}\left[\hat { p } ^ { - 2 } \left\{-I_{1}^{S . P \cdot}(n, 2)+2 I_{1}^{S . P \cdot}(1, n+1)+I_{2}^{S . P .}(n, 2)\right.\right. \\
& -2 I_{2}^{S . P \cdot}(1, n+1)-2 I_{3}^{S . P .}(n+1,1)+2 I_{4}^{S . P .}(n+2,0)-2 I_{6}^{S . P \cdot}(n+2,0) \\
& \left.+2 I_{8}^{S . P .}(n, 2)\right\}+\hat{p}^{-1}\left\{I_{1}^{S . P .}(n, 1)-I_{1}^{S . P \cdot}(0, n+1)-I_{2}^{S . P .}(n, 1)+2 I_{2}^{S . P .} \cdot(1, n)\right. \\
& \left.-I_{2}^{S . P .}(0, n+1)-2 I_{3}^{S . P \cdot}(n, 1)+2 I_{3}^{S . P .}(n+1,0)\right\}+2 I_{1}^{S . P .}(0, n)-2 I_{15}^{S . P .}(n, 0) \\
& +\hat{p}^{-1}\left\{I_{1}^{D \cdot P \cdot}(n, 1)-I_{1}^{D \cdot P \cdot}(0, n+1)-2 I_{1}^{D \cdot P \cdot}(1, n)-I_{2}^{D . P \cdot}(n, 1)-I_{2}^{D . P \cdot}(0, n+1)\right. \\
& \left.\left.+2 I_{2}^{D \cdot P \cdot}(1, n)+4 I_{6}^{D \cdot P \cdot}(n+1,0)\right\}-I_{1}^{D \cdot P \cdot}(n, 0)+I_{1}^{D \cdot P \cdot}(0, n)+I_{9}^{D \cdot P \cdot}(n, 0)\right] \\
& =-8\left(C_{F}^{2}-\frac{1}{2} C_{F} C_{G}\right) \frac{1}{n+1}\left(S_{1}(n)+\frac{1}{n+1}\right) . \\
& \gamma_{n}^{(j)}=4 C_{F} C_{G} \hat{p}^{-n}\left[2 \hat { p } ^ { - 2 } \left\{I_{1}^{S . P .}(n, 2)-2 I_{1}^{S . P .}(1, n+1)-I_{2}^{S . P .}(n, 2)+2 I_{2}^{S . P .}(1, n+1)\right.\right. \\
& \left.+2 I_{3}^{S . P .}(n+1,1)-2 I_{4}^{S . P .}(n+2,0)+2 I_{6}^{S . P \cdot}(n+2,0)-2 I_{8}^{S . P .}(n, 2)\right\} \\
& +\hat{p}^{-1}\left\{-3 I_{1}^{S . P \cdot}(n, 1)+3 I_{1}^{S . P \cdot}(0, n+1)+3 I_{2}^{S . P \cdot}(n, 1)-4 I_{2}^{S . P \cdot}(1, n)+I_{2}^{S . P \cdot}(0, n+1)\right. \\
& \left.+4 I_{3}^{S . P .}(n, 1)-4 I_{3}^{S . P \cdot}(n+1,0)\right\}+I_{1}^{S . P .}(n, 0)-3 I_{1}^{S . P .}(0, n)+4 I_{15}^{S . P .}(n, 0) \\
& +\hat{p}^{-1}\left\{-I_{1}^{D \cdot P \cdot}(n, 1)+I_{1}^{D \cdot P \cdot}(0, n+1)+4 I_{1}^{D \cdot P \cdot}(1, n)+I_{2}^{D \cdot P \cdot}(n, 1)+3 I_{2}^{D \cdot P \cdot}(0, n+1)\right. \\
& \left.\left.-4 I_{2}^{D \cdot P \cdot}(1, n)-8 I_{6}^{D \cdot P \cdot}(n+1,0)\right\}+I_{1}^{D \cdot P \cdot}(n, 0)-3 I_{1}^{D \cdot P \cdot}(0, n)-I_{9}^{D \cdot P \cdot}(n, 0)\right] \\
& =0 \text {. }
\end{aligned}
$$

The contribution from $(\mathrm{j})$ turned out to be identically zero for all $n$.

$$
\begin{aligned}
& \gamma_{n}^{(k)}=8\left(C_{F}^{2}-\frac{1}{2} C_{F} C_{G}\right) \hat{p}^{-n}\left[\hat { p } ^ { - 2 } ( 1 + ( - 1 ) ^ { n } ) \left\{I_{3}^{S . P .}(n+2,0)-2 I_{8}^{S . P .}(n, 2)-2 I_{16}^{S . P .}(n, 2)\right.\right. \\
& \left.+4 I_{16}^{S . P .}(n+1,1)-2 I_{16}^{S . P .}(n+2,0)\right\}+\hat{p}^{-1}\left\{I_{2}^{S . P \cdot}(n, 1)+I_{12}^{S . P .} \cdot(n, 1)-I_{12}^{S . P .}(n+1,0)\right. \\
& -I_{13}^{S . P .}(n, 1)+I_{13}^{S . P \cdot}(n+1,0)-I_{14}^{S . P .}(n, 1)+4\left(1+(-1)^{n}\right) I_{16}^{S . P .}(n, 1) \\
& \left.-4\left(1+(-1)^{n}\right) I_{16}^{S . P .}(n+1,0)\right\}+\left(1+(-1)^{n}\right) I_{3}^{S . P .}(n, 0)-(-1)^{n} I_{12}^{S . P .}(n, 0)+2 I_{13}^{S . P .}(n, 0) \\
& +I_{14}^{S . P \cdot}(n, 0)-2\left(1+(-1)^{n}\right) I_{16}^{S . P \cdot}(n, 0)+\hat{p}^{-1}\left\{I_{2}^{D \cdot P \cdot}(n, 1)+I_{12}^{D \cdot P \cdot}(n, 1)-I_{12}^{D . P \cdot}(n+1,0)\right.
\end{aligned}
$$




$$
\begin{aligned}
& \left.\left.-I_{13}^{D \cdot P \cdot}(n, 1)+I_{13}^{D \cdot P \cdot}(n+1,0)-I_{14}^{D \cdot P \cdot}(n, 1)\right\}-I_{11}^{D \cdot P \cdot}(n, 0)+I_{13}^{D \cdot P \cdot}(n, 0)+I_{14}^{D \cdot P \cdot}(n, 0)\right] \\
= & -8\left(C_{F}^{2}-\frac{1}{2} C_{F} C_{G}\right)\left(1+(-1)^{n}\right)\left(\frac{1}{n+1}-\frac{1}{n+2}\right) .
\end{aligned}
$$

This result is zero for odd- $n$. This can be easily proved by assigning the momenta as shown in Fig. 10. Then the denominator is symmetric under $k_{1} \leftrightarrow k_{2}$. The numerator has a factor $\left(\hat{k}_{1}-\hat{k}_{2}\right)^{n}$. It is convenient to consider the sum of Fig. 10 and its charge conjugation. Then one can easily show the contribution from (k) becomes 0 for odd- $n$ with the use of lemma in section 3.

$$
\begin{aligned}
& \gamma_{n}^{(n)}=8 C_{F} C_{G} \hat{p}^{-n} \sum_{l=1}^{n}\left[\hat { p } ^ { - 1 } \left\{-I_{1}^{S . P .}(l-1, n-l+2)+I_{1}^{S . P .}(l, n-l+1)\right.\right. \\
& \left.\left.+I_{2}^{S . P .}(l-1, n-l+2)-I_{2}^{S \cdot P \cdot}(l, n-l+1)\right\}+I_{1}^{S . P .}(l, n-l)\right] \\
& =4 C_{F} C_{G}\left[S_{3}(n)-2 \widehat{S}(n)-\frac{S_{1}^{2}(n)}{2(n+1)}-\frac{S_{2}(n)}{2(n+1)}+\frac{(2 n-1) S_{1}(n)}{(n+1)^{2}}+\frac{2 n}{(n+1)^{3}}\right] . \\
& \gamma_{n}^{(o)}=-16\left(C_{F}^{2}-\frac{1}{2} C_{F} C_{G}\right) \hat{p}^{-n} \sum_{l=1}^{n}\left[\hat { p } ^ { - 1 } \left\{-I_{2}^{S \cdot P \cdot}(n-l, l+1)+I_{2}^{S . P \cdot}(n-l+1, l)\right.\right. \\
& +I_{12}^{S . P .} \cdot(n-l, l+1)-2 I_{12}^{S . P .} \cdot(n-l+1, l)+I_{12}^{S . P .} \cdot(n-l+2, l-1) \\
& -I_{13}^{S . P .}(n-l, l+1)+2 I_{13}^{S . P .}(n-l+1, l)-I_{13}^{S . P .}(n-l+2, l-1) \\
& \left.+I_{14}^{S . P .}(n-l, l+1)-I_{14}^{S . P \cdot}(n-l+1, l)\right\}+I_{13}^{S . P .}(n-l, l)-I_{13}^{S . P .}(n-l+1, l-1) \\
& \left.-I_{14}^{S . P .}(n-l, l)-I_{14}^{S . P .}(n-l+1, l-1)\right] \\
& =-8\left(C_{F}^{2}-\frac{1}{2} C_{F} C_{G}\right)\left[4\left(S_{1}(n)+\frac{1}{n+1}\right)\left(S_{2}^{\prime}\left(\frac{n}{2}\right)-S_{2}(n)\right)-4 \widehat{S}(n)-8 \widetilde{S}(n)\right. \\
& \left.+2 S_{3}(n)+S_{3}^{\prime}\left(\frac{n}{2}\right)-\frac{2 S_{1}(n)}{n+1}+\frac{4\left(1-(-1)^{n}\right)}{(n+1)^{3}}-\frac{2 n}{(n+1)^{3}}\right] .
\end{aligned}
$$

In the above expression, we introduced the function

$$
\widehat{S}(n)=\sum_{j=1}^{n} \frac{1}{j^{2}} S_{1}(j) .
$$




\section{References}

[1] J.P. Ralston and D.E. Soper, Nucl. Phys. B152, 109 (1979).

[2] X. Artru and M. Mekhfi, Z. Phys. C45 669 (1990); Nucl. Phys. A532 (1991) 351c.

[3] R.L. Jaffe and X. Ji, Phys. Rev. Lett. 67, 552 (1991); Phys. Rev. Lett 71, 2547 (1993).

[4] J.L. Cortes, B. Pire and J.P. Ralston, Z. Phys. C55, 409 (1992).

[5] H. Georgi and H.D. Politzer, Phys. Rev. D9, 416 (1974). D. J. Gross and F. Wilczek, Phys. Rev. D9, 980 (1974); G. A. Altarelli and G. Parisi, Nucl. Phys. B126, 298 (1977).

[6] Y. Koike and K. Tanaka, Phys. Rev. D51, 6125 (1995).

[7] E.G. Floratos, D.A. Ross and C.T. Sachrajda, Nucl. Phys. B129, 66 (1977); B139, 545 (1978) (E).

[8] E.G. Floratos, D.A. Ross and C.T. Sachrajda, Nucl. Phys. B152, 493 (1979).

[9] A. Gonzalez-Arroyo, C. Lopez and F.J. Yndurain, Nucl. Phys. B153, 161 (1979).

[10] E.G. Floratos, R. Lacaze and C. Kounnas, Phys. Lett. 98B, 89 (1981); 285 (1981).

[11] G. Curci, W. Furmanski and R. Petronzio, Nucl. Phys. B175, 27 (1980).

[12] W. Furmanski and R. Petronzio, Phys. Lett 97B, 437 (1980).

[13] R. Mertig, W.L. van Neerven, Z. Phys. C70 637 (1996).

[14] W. Vogelsang, Phys. Rev. D54 2023 (1996).

[15] M. Glück, E. Reya and A. Vogt, Z phys. C53, 127 (1992); A. D. Martin, W.J. Stirling and R.G. Roberts, Phys. Lett. B354, 155 (1995); H.L. Lai et al. Phys. Rev. D51, 4763 (1995).

[16] M. Glück, E. Reya, M. Stratmann and W. Vogelsang, Phys. Rev. D53, 4775 (1996); T. Gehrmann and W.J. Stirling, Phys. Rev. D53, 6100 (1996); G. Altarelli, R. Ball, S. Forte and G. Ridolfi, hep-ph/9701289.

[17] W. Vogelsang, hep-ph/9706511.

[18] S. Kumano and M. Miyama, hep-ph/9706420. 


\section{Figure captions}

Fig. 1 Basic vertices for $O_{n}^{\mu} \cdot \Delta$ with 0,1 and 2 gluon lines.

Fig. 2 Two-loop corrections to the one-particle irreducible two-point Green function which imbeds the operator $O_{n}^{\mu} \cdot \Delta$. For diagrams not symmetric, crossed diagrams must be included. In Figs. (f) and (h), the contributions from the Faddev-Popov ghost and tadpole diagram must be added.

Fig. 3 The same as Fig. 2 but for the flavor-singlet distribution. These diagrams become identically zero for $h_{1}$.

Fig. 4 Two-loop corrections to the quark self-energy. The ghost-loop and the tadpole contribution is implied as in Fig. 2.

Fig. 5 Momentum labeling for the diagram Fig. 2(i).

Fig. 6 The counter term contributions to Fig. 2(i).

Fig. 7 The coefficients of the two-loop anomalous dimension $\gamma_{n}^{f, h(1)}$ for $N_{f}=3$ (circle) and $N_{f}=5$ (triangle).

Fig. 8 (a) $\gamma_{n}^{f, h(1)} / 2 \beta_{1}$ (circle) and $\gamma_{n}^{f, h(0)} / 2 \beta_{0}$ (square). (b) $\gamma_{n}^{f, h(1)} / 2 \beta_{1}-\gamma_{n}^{f, h(0)} / 2 \beta_{0}$ for $N_{f}=$ 3,5 .

Fig. 9 (a) The LO and NLO $Q^{2}$-evolution of the tensor charge normalized at $Q^{2}=1 \mathrm{GeV}^{2}$. (b) The LO and NLO $Q^{2}$-evolution of the first moment of $h_{1}\left(x, Q^{2}\right)$ and $f_{1}\left(x, Q^{2}\right)$ normalized at $Q^{2}=1 \mathrm{GeV}^{2}$.

Fig. 10 Momentum labeling for the diagram Fig. 2(k). 

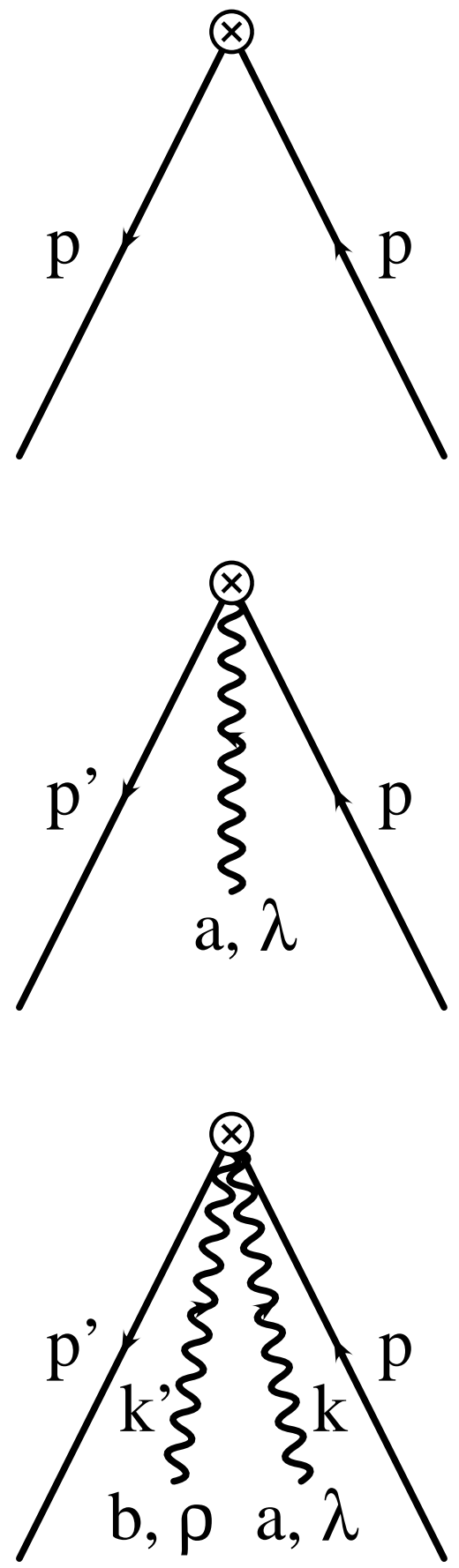

Fig.1 


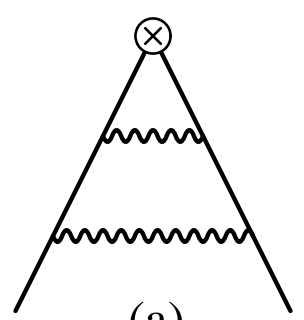

(a)

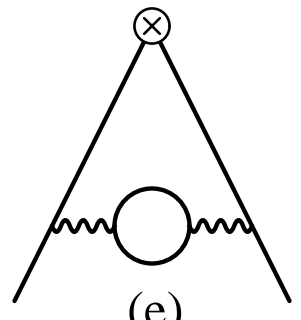

(e)

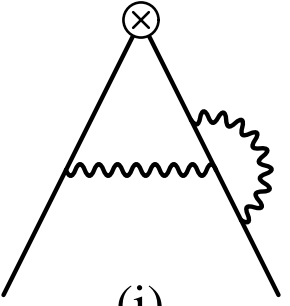

(i)

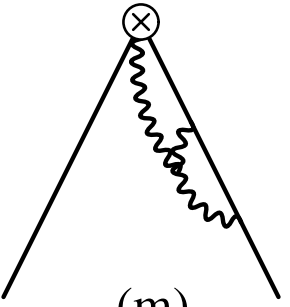

(m)

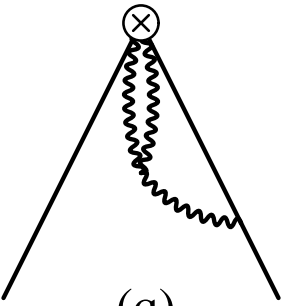

(q)

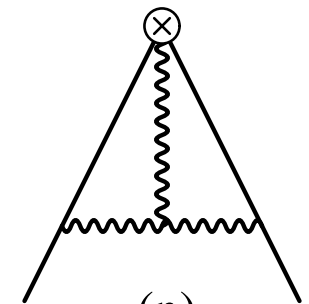

(n)

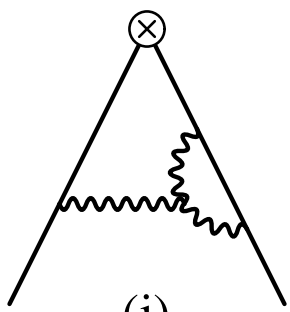

(j)

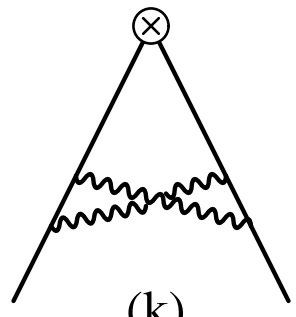

(k)

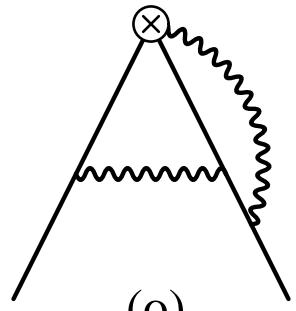

(o)

(c)

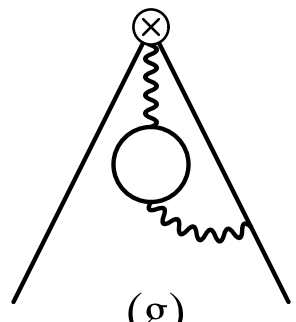

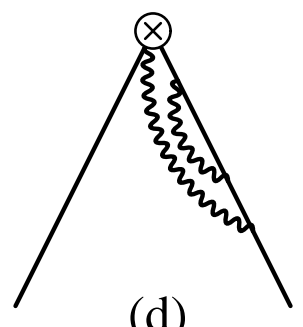

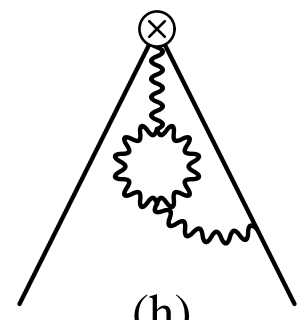

(h)

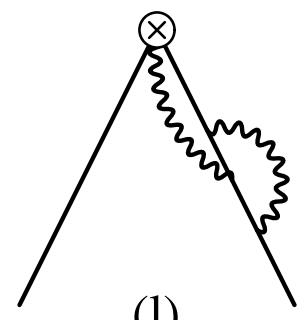

(1)

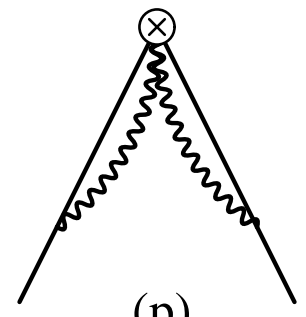

(p)

Fig.2 

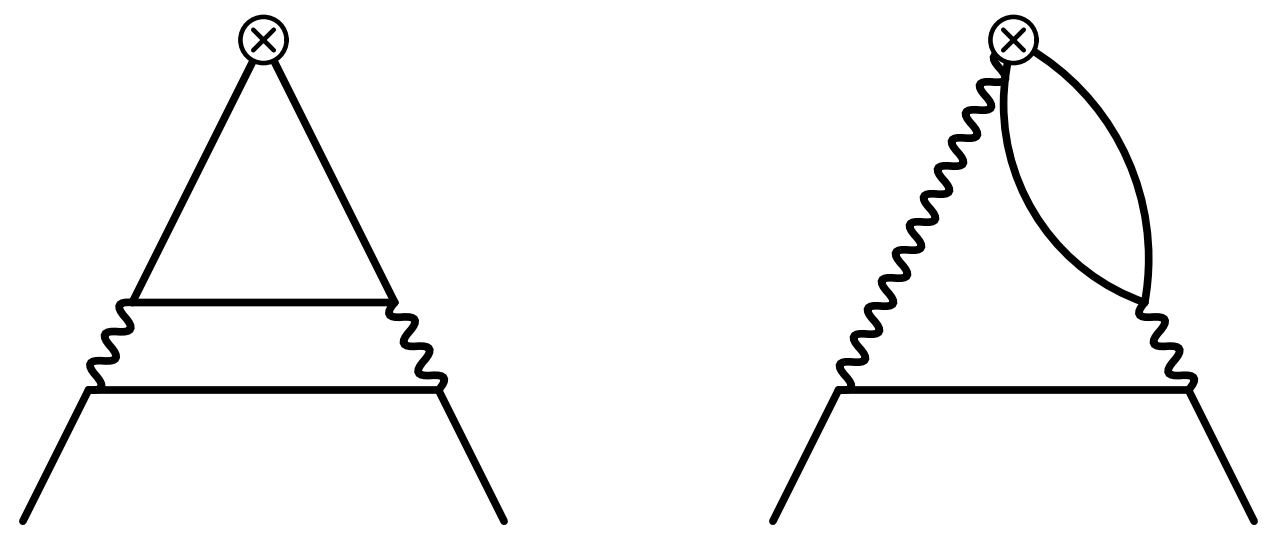

Fig. 3 

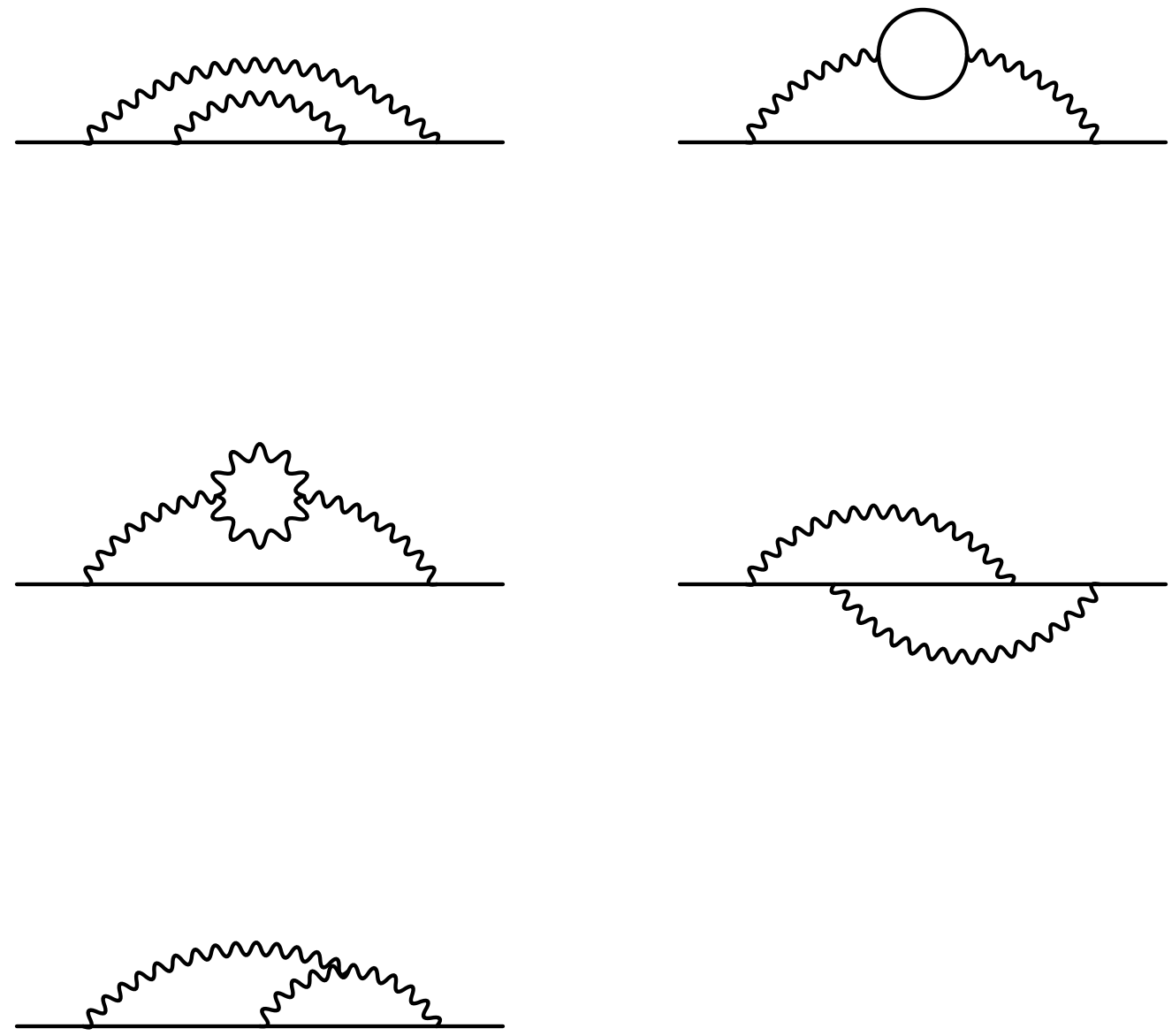

Fig.4 


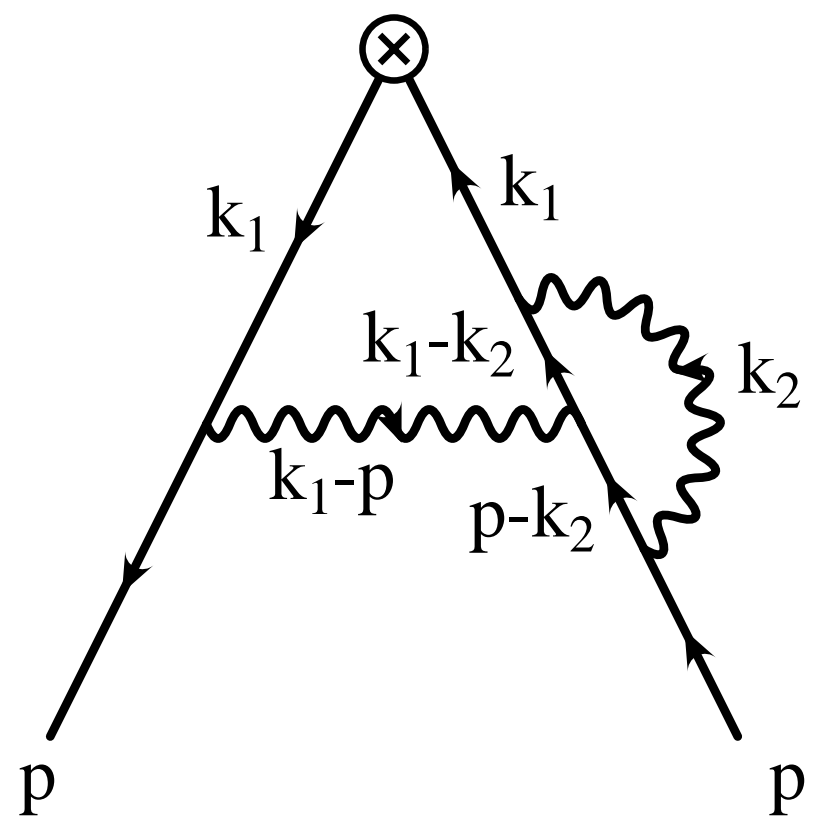

(i)

Fig.5 

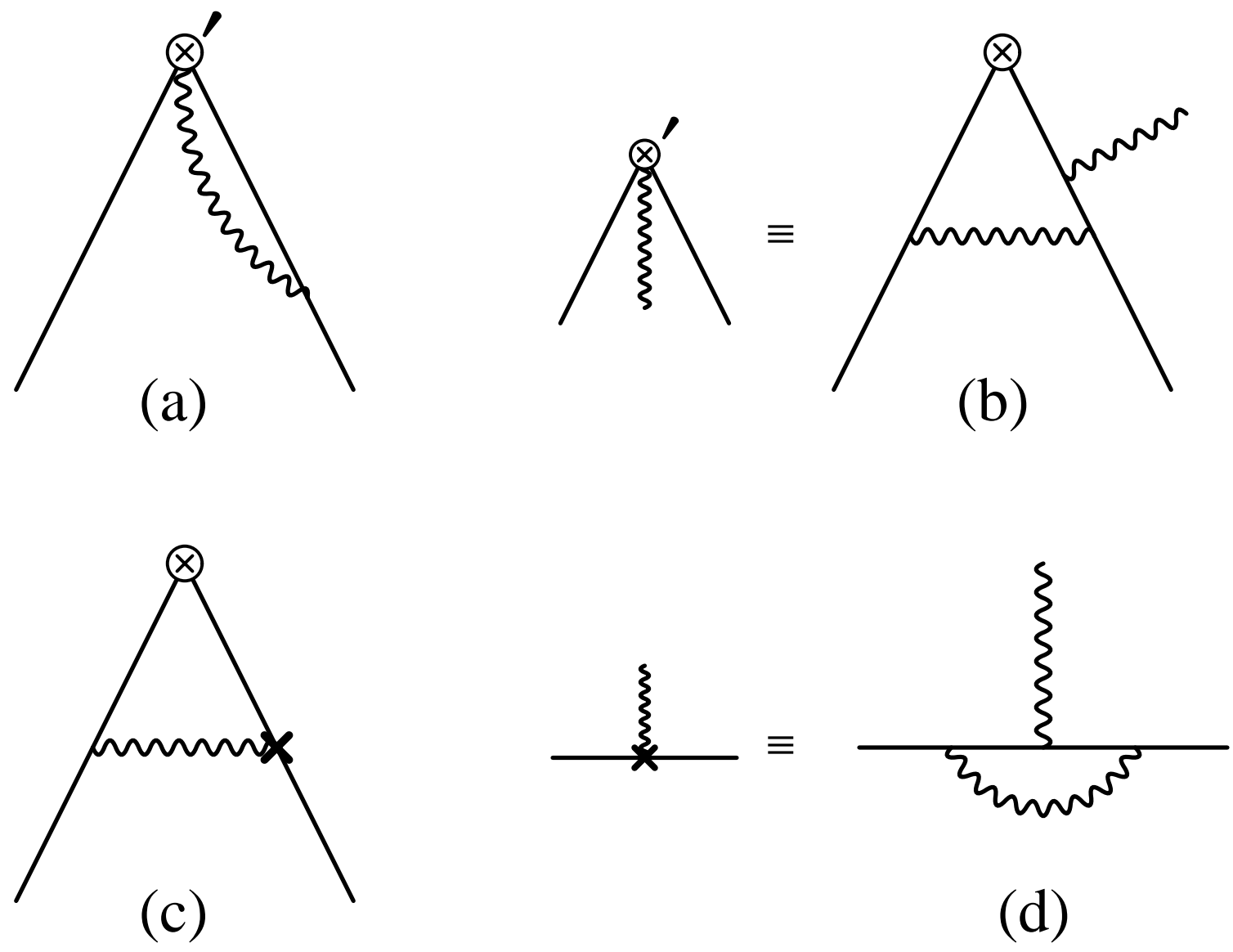

Fig.6 


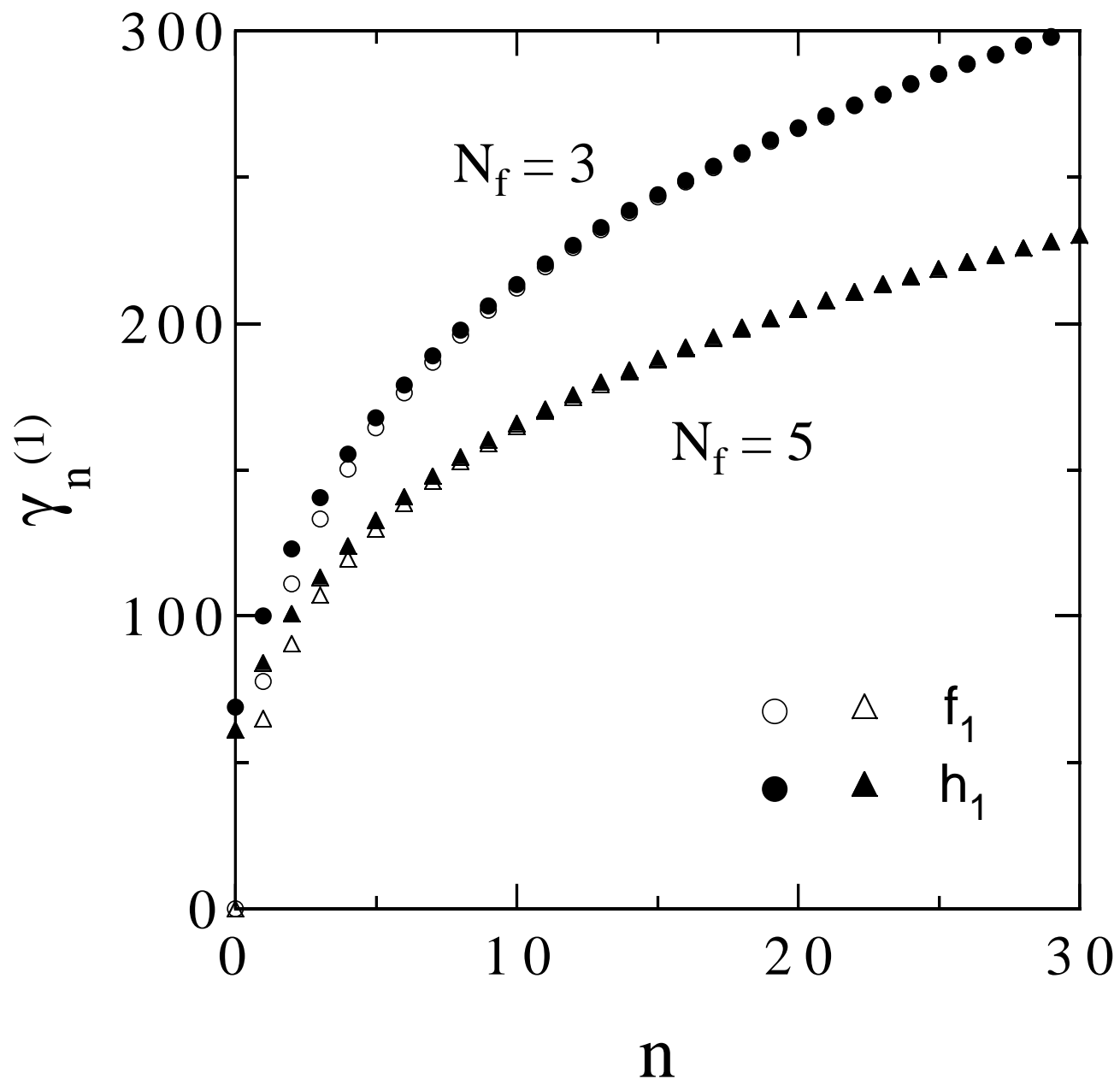

Fig.7 

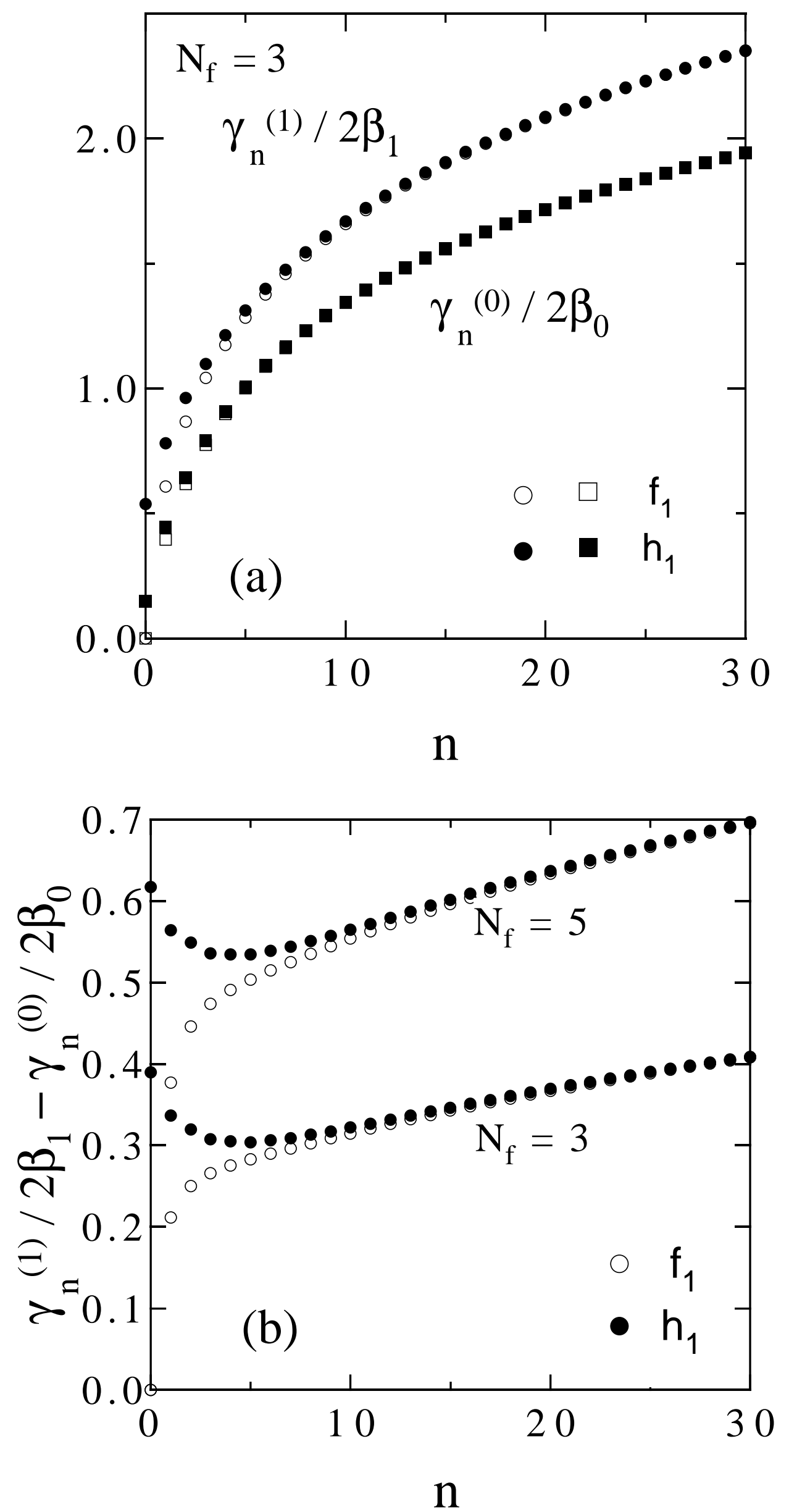

Fig.8 

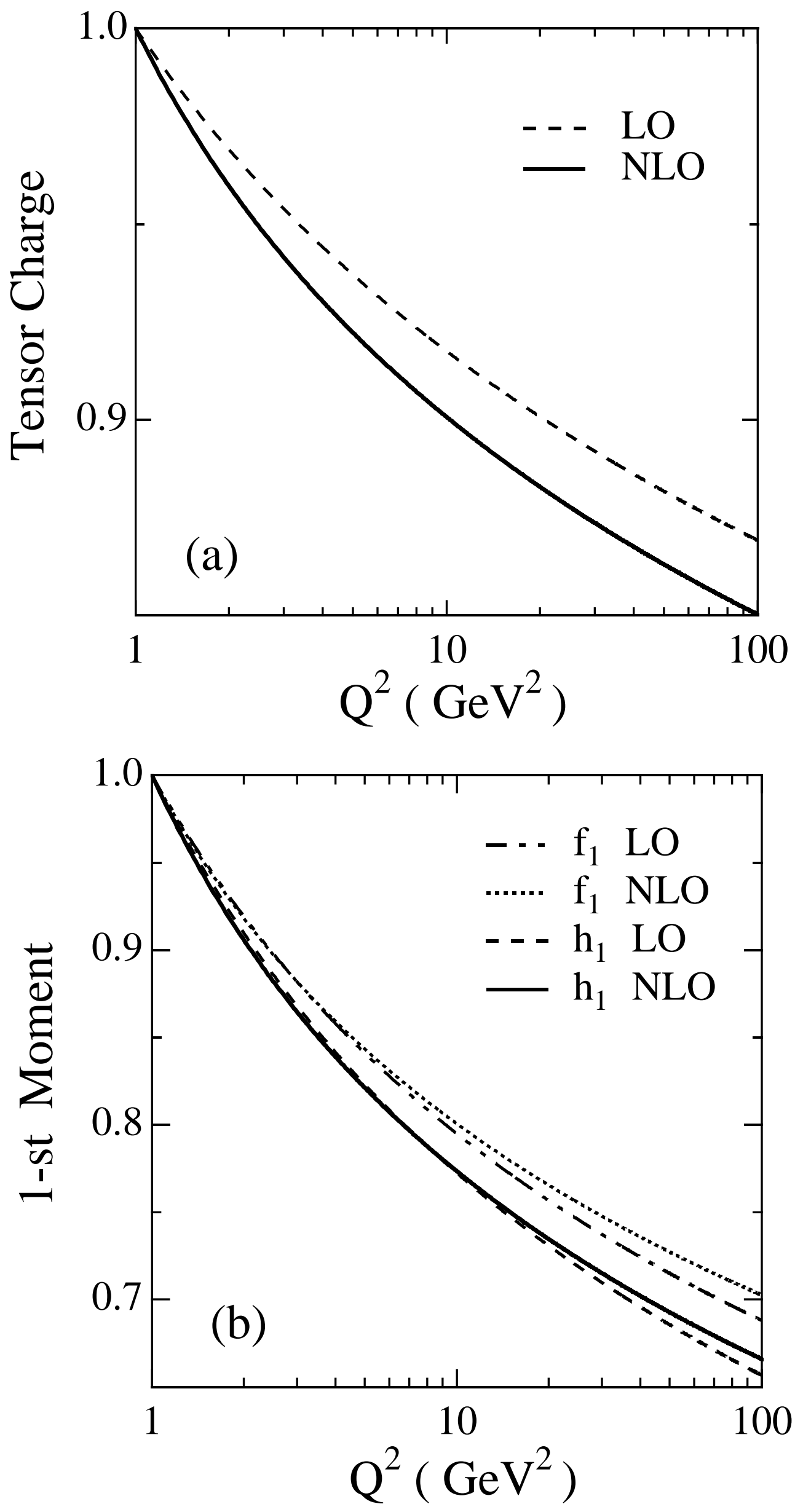

Fig.9 


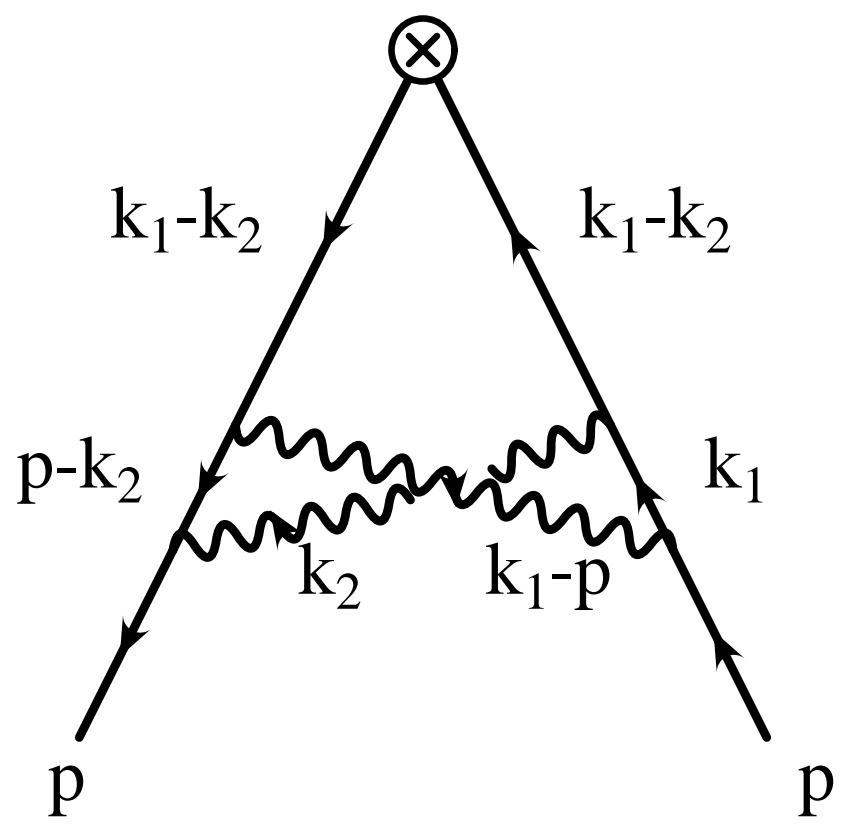

(k)

Fig.10 